\title{
Vaccine-modified NF-kB and GR signaling in cervicovaginal epithelium correlates with protection
}

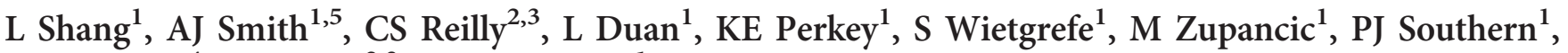 \\ RP Johnson ${ }^{4}$, JV Carlis ${ }^{2,3}$ and AT Haase ${ }^{1}$
}

Cervicovaginal epithelium plays a critical role in determining the outcome of virus transmission in the female reproductive tract (FRT) by initiating or suppressing transmission-facilitating mucosal immune responses in naïve and SIVmac239Anef-vaccinated animals, respectively. In this study, we examined the very early responses of cervical epithelium within $24 \mathrm{~h}$ after vaginal exposure to SIV in naive and SIVmac239Anef-vaccinated rhesus macaques. Using both ex vivo and in vivo experimental systems, we found that vaginal exposure to SIV rapidly induces a broad spectrum of pro-inflammatory responses in the epithelium associated with a reciprocal regulation of NF-kB and glucocorticoid receptor (GR) signaling pathways. Conversely, maintenance of high-level GR expression and suppression of NF-kB expression in the epithelium were associated with an immunologically quiescent state in the FRT mucosa and protection against vaginal challenge in SIVmac239 $\Delta$ nef-vaccinated animals. We show that the immunologically quiescent state is induced by FCGR2B-immune complexes interactions that modify the reciprocal regulation of NF-kB and GR signaling pathways. Our results suggest that targeting the balance of NF-kB and GR signaling in early cervicovaginal epithelium responses could moderate mucosal inflammation and target cell availability after vaginal infection, thereby providing a complementary approach to current prevention strategies.

\section{INTRODUCTION}

Every year more than 2 million people are newly infected with $\mathrm{HIV},{ }^{1}$ mainly by sexual transmission. Women are particularly vulnerable, currently accounting for $\sim 60 \%$ of newly acquired infections. ${ }^{2}$ Thus, effective strategies for preventing HIV-1 sexual transmission to women are urgently needed.

To that end, we have been investigating HIV-1 heterosexual transmission to women in the Simian Immunodeficiency Virus (SIV)-rhesus macaque model for insights that could guide development of effective preventive vaccines and microbicides. ${ }^{3-10}$ In this model, we have discovered two critical events in mucosal transmission: ${ }^{3,5}$ (1) early establishment of founder populations of infected cells; and (2) local expansion of virus infection fueled by the recruitment of CD4 T cells into cervicovaginal mucosa that subsequently disseminates and establishes a robust systemic infection. More recently, we have shown that vaginal exposure to the SIV inoculum elicits changes in epithelial responses that affect both of these critical transmission-facilitating events. ${ }^{3,6}$ Vaginal exposure to SIV rapidly induces stress responses in the cervicovaginal epithelial cells that are associated with disruption of epithelium integrity and loss of epithelium barrier functions, thus opening a route for virus to infect mucosal CD4 $\mathrm{T}$ cells and establish infected founder populations (manuscript in preparation). Moreover, vaginal exposure to SIV inoculum also quickly triggers production of pro-inflammatory chemokines in the female reproductive tract (FRT) epithelium, subsequently leading to the recruitment of macrophages and plasmacytoid dendritic cells (pDCs), which in turn generates a gradient of chemokine concentration to recruit more CD4 T cells to fuel local expansion. ${ }^{6}$

${ }^{1}$ Department of Microbiology and Immunology, Medical School, University of Minnesota, Minneapolis, Minnesota, USA. ${ }^{2}$ Division of Biostatistics, School of Public Health, University of Minnesota, Minneapolis, Minnesota, USA. ${ }^{3}$ Department of Computer Science and Engineering, University of Minnesota, Minneapolis, Minnesota, USA and ${ }^{4}$ Yerkes National Primate Research Center, Emory University, Atlanta, Georgia, USA. Correspondence: AT Haase (haase001@umn.edu)

${ }^{5}$ Current address: Bridge-to-MD Program, One Battery Park Plaza, New York, NY 10004, USA. 


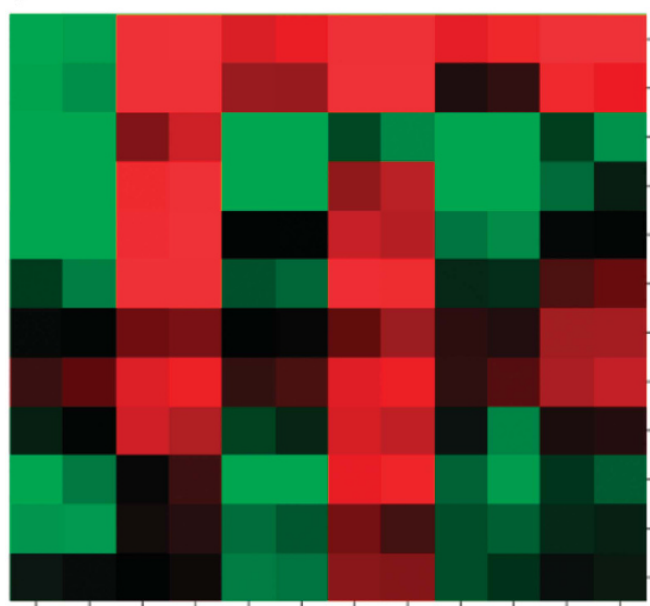

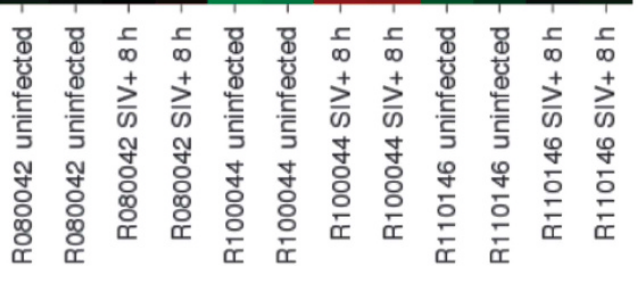

b

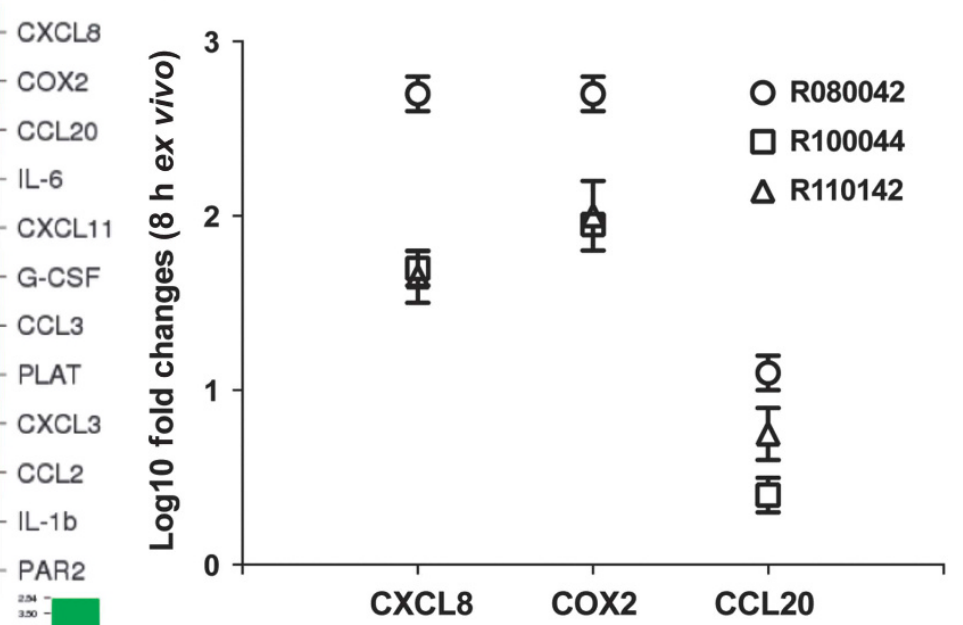

c

CXCL8

cox-2

CCL20

IL-6
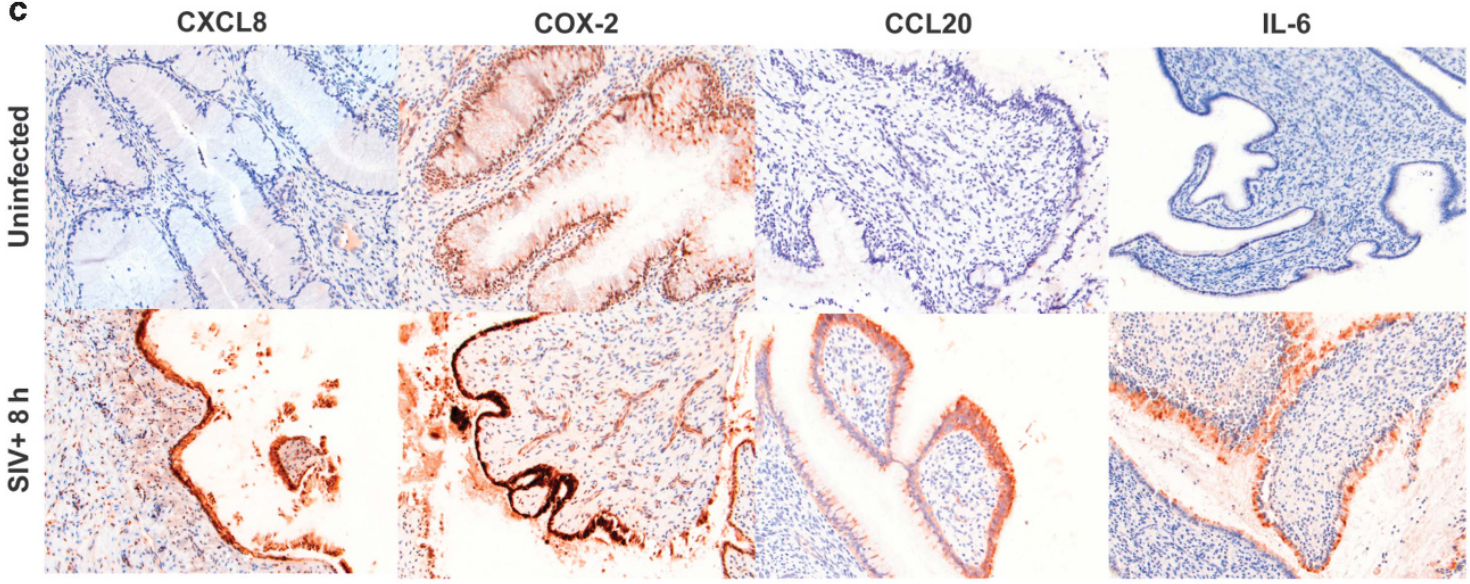

G-CSF

CCL3

CXCL3

IL-1b

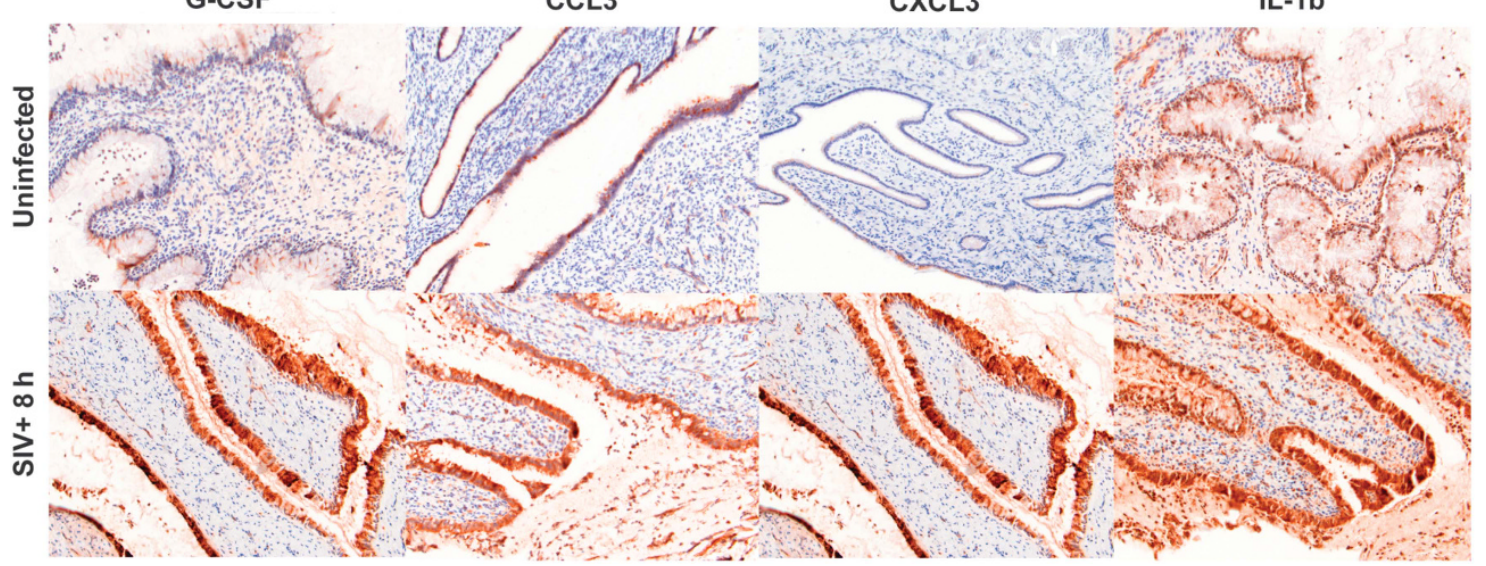

Figure 1 Continued. 
d

$\operatorname{cox}-2$

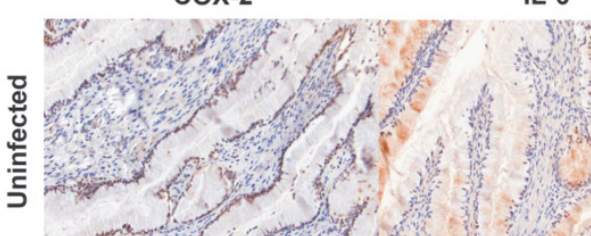

CXCL3

IL-1b
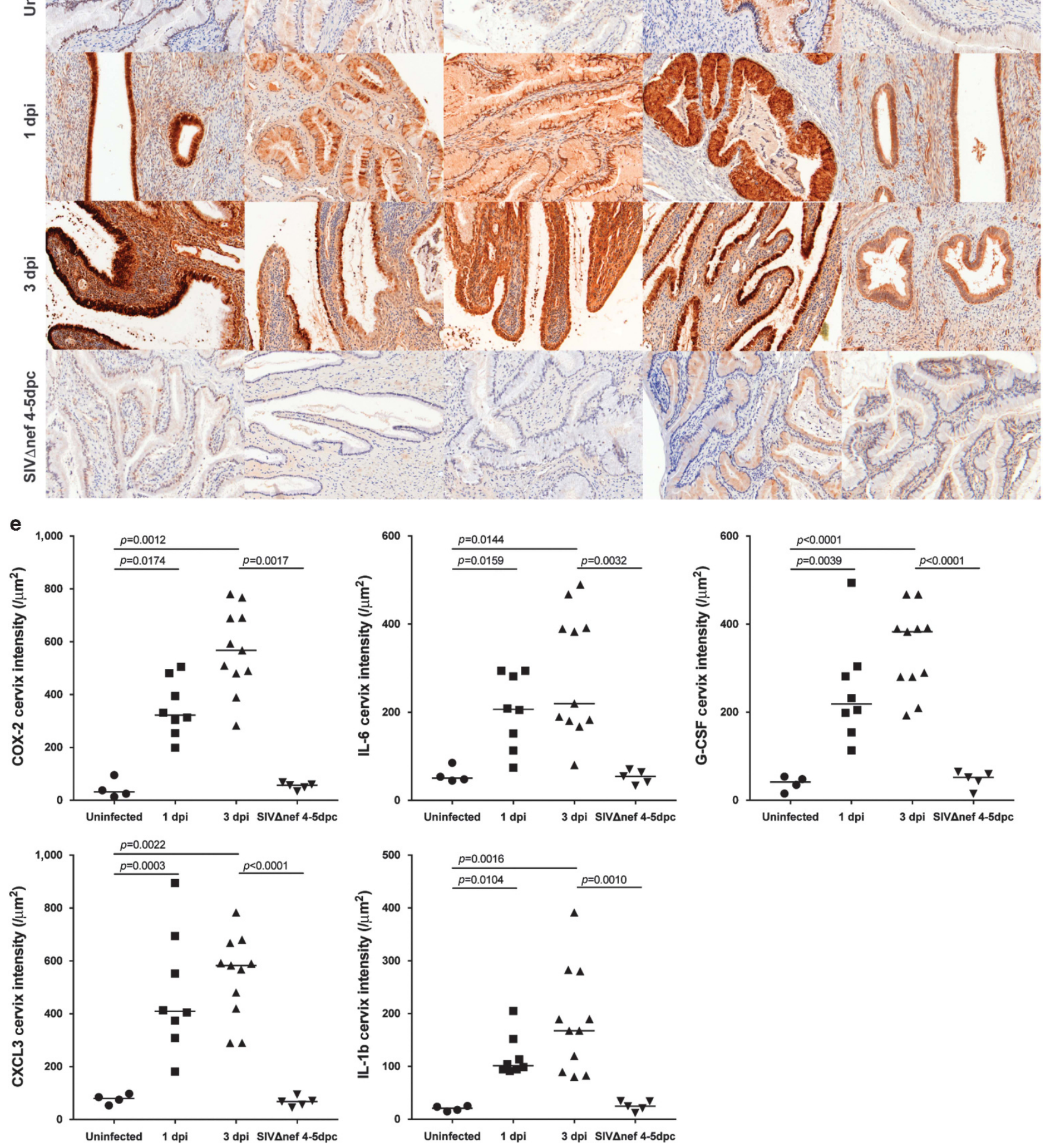

Figure 1 Continued. 
The cervicovaginal epithelium also plays a critical role in protection against vaginal transmission conferred by the live attenuated virus vaccine, SIVmac239 $\Delta$ nef (SIV $\Delta$ nef) in two ways. ${ }^{4,6,8,9}$ First, SIV $\Delta$ nef-vaccination induces production of antibodies (Abs) reactive to trimeric gp41 in the circulation and mucosal tissues. Local production of these Abs in spatial proximity to neonatal $\mathrm{Fc}$ receptor $(\mathrm{FcRn})$-expressing cervical and vaginal epithelium generates high local concentrations of Abs to block entry and establishment of infected founder populations at the portal of entry. Second, the FcRnconcentrated Abs form immune complexes (IC) with incoming virus at mucosal front lines. These IC interact with the inhibitory Fc receptor, FCGR2B, which induces a suppressive signaling program in cervicovaginal epithelial cells to block the early chemokine production in FRT epithelium and the subsequent CD4 T-cell recruitment that underpins local expansion of infection.

Here we delve more deeply into how cervicovaginal epithelium responses are regulated in SIV $\Delta$ nef-vaccinated and unvaccinated animals at the intracellular signaling level. In this study, we combined microarray analysis and immunohistochemical tissue mapping of the very early responses in cervicovaginal epithelium to virus exposure. We found that the distinct responses to virus exposure in cervicovaginal epithelium of SIV $\Delta$ nef-vaccinated and unvaccinated animals is correlated with differential regulation of the NF-kB and glucocorticoid receptor (GR) signaling pathways.

\section{RESULTS}

\section{Ex vivo and in vivo analysis of the cervical epithelial response to SIV exposure in naïve and $\operatorname{SIV} \Delta$ nef-vaccinated animals}

We analyzed the immediate responses of epithelium to SIV by topically exposing fresh cervical explants of RMs to pathogenic SIVmac251 for $8 \mathrm{~h}$, and examined the global transcription profiles of epithelium-enriched mucosal tissues by microarray analysis. Having identified genes that immediately responded to topical exposure, we then mapped their expression in the tissues by immunohistochemistry. The advantages of this ex vivo approach are (1) the immediate response of cervical epithelium to viral exposure can be examined; and (2) tissues from the same individual animal can be compared pairwise with and without infection, thereby minimizing noise from inter-animal variation, a useful tactic with a small group of animals. We then compared the results from the ex vivo analysis to those from the examination of archived tissues that had been collected at necropsy from naïve or
SIV $\Delta$ nef-vaccinated animals after vaginal challenge with high doses of SIV.

\section{SIV vaginal exposure rapidly induces epithelial expression of pro-inflammatory chemokines, cytokines, and other inflammatory mediators in naïve animals, but not in SIV $\Delta$ nef-vaccinated animals}

In the microarray analysis of mucosal tissues "challenged" with SIV ex vivo, there were significant increases in the transcription of host genes in three categories involved in promoting proinflammatory responses (Figure 1a,b). First, six proinflammatory chemokine ligands were quickly induced $8 \mathrm{~h}$ after exposure to SIV, including CCL2, CCL3, CCL20, CXCL3, CXCL8, and CXCL11 (Figure 1a). Second, expression of proinflammatory cytokines IL-1 $\beta$, IL-6, and granulocyte colonystimulating factor also increased (Figure 1a). Third, viral exposure enhanced the transcription of some key genes involved in inflammation as well, such as COX-2, PAR2, and PLAT (Figure 1a). By immunohistochemistry, increased expression of these genes mapped predominantly and strikingly to cervical epithelium (Figure 1c). In vivo, in the necropsy tissues from cohorts of vaginally infected/unvaccinated RMs, we found increased endocervical (but not ectocervical) epithelial expression of the same spectrum of genes within 1-3 dpi (Figure 1d), supporting the validity of the ex vivo culture system as a model to mimic early responses of the cervical epithelium in vivo. By contrast, we did not detect increased epithelial expression of these genes in the cervix of SIV $\Delta$ nef-vaccinated animals at 4-5 dpc, the earliest time point available for archived tissues of SIV $\Delta$ nef-vaccinated animals after vaginal challenge (Figure 1d; quantified in Figure 1e), consistent with previously reported evidence of an immunologically "quiescent" state of the epithelium in vaccinated animals. ${ }^{3}$

\section{Dynamic changes in NF-kB and other signaling pathways in the early epithelial responses to SIV}

To identify potential signaling pathways that might be involved in these early epithelial responses to SIV, we screened the microarray results from the ex vivo analysis for key signaling molecules and transcription factors (TFs) in the common signaling pathways of innate immune responses and inflammation. We included: (1) key signaling molecules in the NF-kB, MAPK, JAK-STAT, IRF, PI3K-AKT, PLC-PKC/Ca2+, cAMP-PKA, and inflammasome pathways; and (2) TFs of the NF-kB, AP-1, IRF, ATF/CREB, and c/EBP families. We found that exposure to SIV ex vivo rapidly activates the NF-kB, IRF1, and cAMP-PKA signaling pathways in cervical tissues (Figure 2a). The particularly noteworthy transcriptional

Figure 1 SIV exposure induces epithelial expression of pro-inflammatory chemokines, cytokines, and other inflammatory mediators in naïve but not SIV $\Delta$ nef-vaccinated animals. (a) The global transcriptional profiles of cervical explants of rhesus macaques after $8 \mathrm{~h}$ topical exposure to SIV ex vivo were examined by microarray analysis and compared pairwise between naïve vs. SIV-exposed tissues from the same animal. Topical SIV exposure rapidly increased cervical expression of a spectrum of pro-inflammatory chemokines, cytokines, and other inflammatory mediators as described under Results section. (b) Log10 fold changes of selected genes detected by microarray analysis were measured by quantitative real time PCR. (c) Images of increased expression ex vivo of some of the chemokines, cytokines, and inflammatory mediators in cervical epithelium at $8 \mathrm{~h}$ shown in $1 \mathrm{a}$ in descending order. (d) Increased expression in vivo in cervix of the same chemokines, cytokines, and inflammatory mediators following vaginal challenge in naïve animals but not SIV $\Delta$ nef-vaccinated animals. (e) Quantification of expression levels by quantitative image analysis of immunohistochemistry-stained in vivo tissues. 
changes included (1) increased expression of key molecules in NF-kB signaling: NF-kB1p50 and NF-kBp65 (RelA); (2) decreased expression of NF-kB signaling inhibitors: COMMD1, COMMD2, and COMMD10; and (3) increased expression of IRF1, CREM, and ATF3 (Figure 2a). Just as we had seen with pro-inflammatory chemokines/cytokines and inflammatory mediators, dynamic changes in expression of these signaling molecules were predominantly localized to cervical epithelium, illustrated for NF-kB signaling molecules in Figure 2c.

In vivo, we documented parallel dynamic changes in the NF-kB pathway with increased cervical epithelial expression of NF-kBp50 and NF-kBp65 (RelA) and decreased expression of COMMD1 in naïve animals but not SIV $\Delta$ nef-vaccinated animals after vaginal challenge (Figure $3 a$; quantified in $3 b$ ). ATF3 epithelial expression also fit this pattern, but increased expression of CREM did not differ significantly between naïve and vaccinated animals; and IRF1 expression was higher in vaccinated animals compared to naïve animals. Based on these results, we focused our subsequent analysis on signaling molecules in the NF-kB pathway in the epithelium of naive and SIV $\Delta$ nef-vaccinated animals following vaginal SIV exposure.

\section{Role of epithelial GR signaling in altering the response to SIV}

How might the mucosal epithelium activate or suppress these signaling pathways in naïve and $\operatorname{SIV} \Delta$ nef-vaccinated animals? In seeking answers to this question, we re-examined a
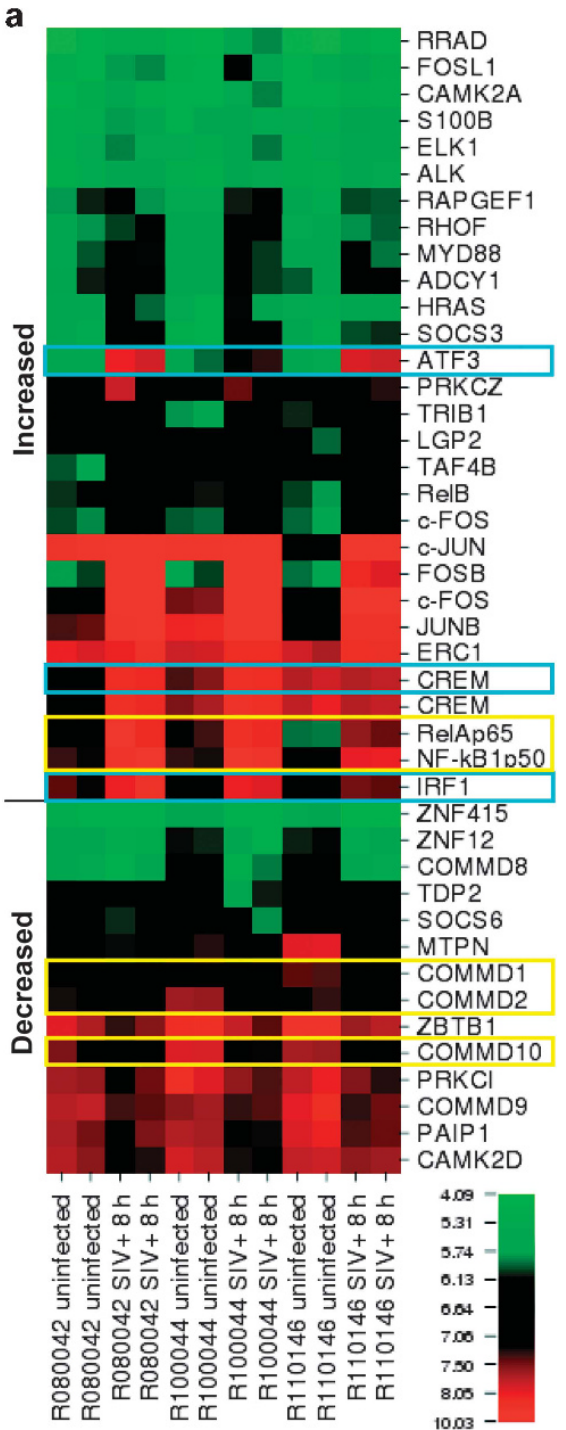

b

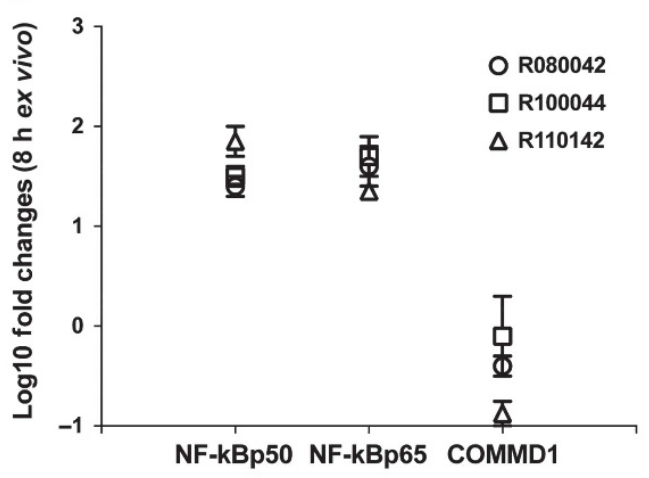

C

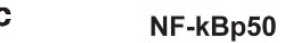

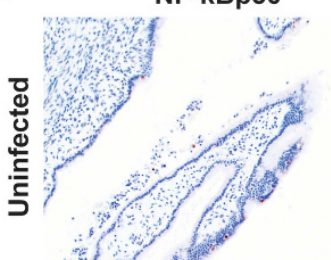

NF-kBp65/RelA

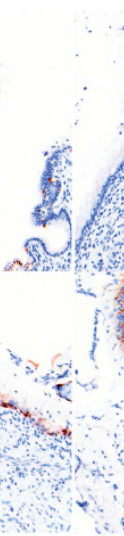

COMMD1

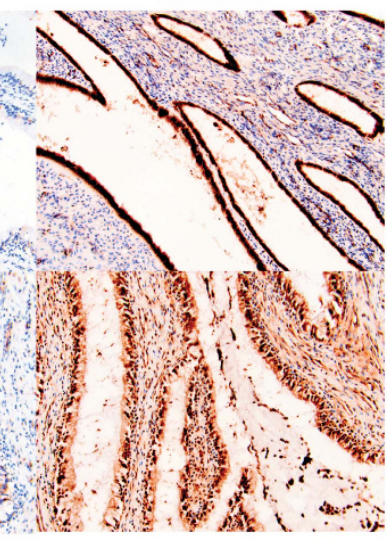

Figure 2 Dynamic changes in NF-kB signaling pathways in early epithelial responses to SIV ex vivo. (a) Microarray analysis ex vivo of cervical explant tissues of rhesus macaques after $8 \mathrm{~h}$ exposure to topical SIV. Increased expression of NF-kB1p50 and NF-kBp65/RelA and decreases in NF-kB signaling inhibitors, COMMD1, COMMD2, and COMMD10 (yellow boxes); increased expression of IRF1 and cAMP-PKA signaling molecules, ATF3 and CREM (blue boxes). (b) Log10 fold changes of selected genes detected by microarray analysis were measured by quantitative real-time PCR. (c) Increased and decreased cervical epithelial expression by immunohistochemical staining of key NF-kB signaling molecules identified by microarray analysis after $8 \mathrm{~h}$ exposure to SIV. 
a

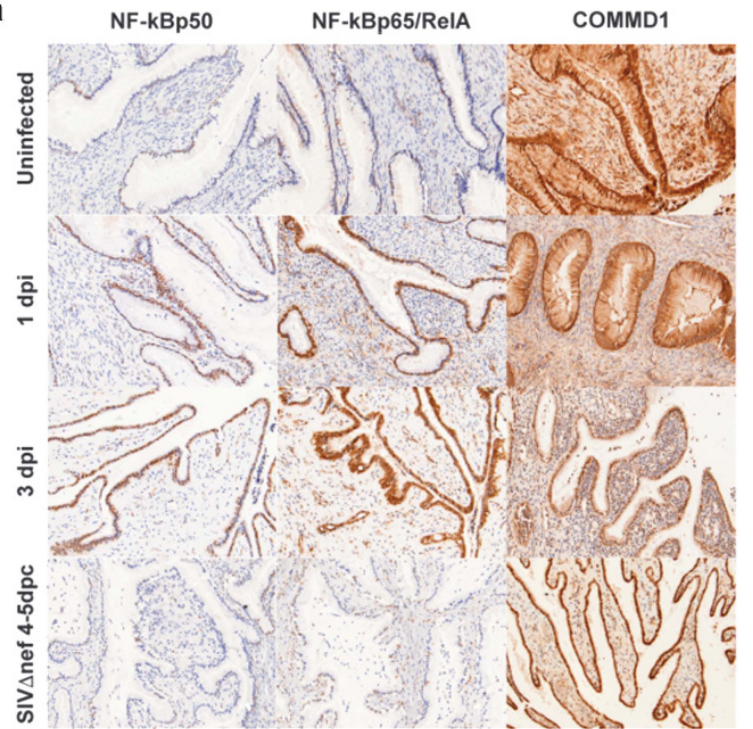

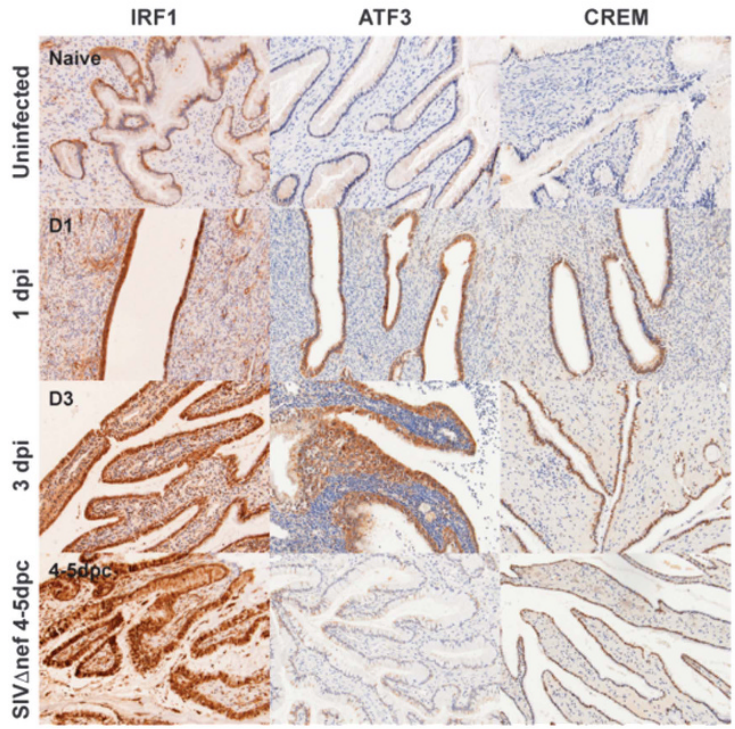

b
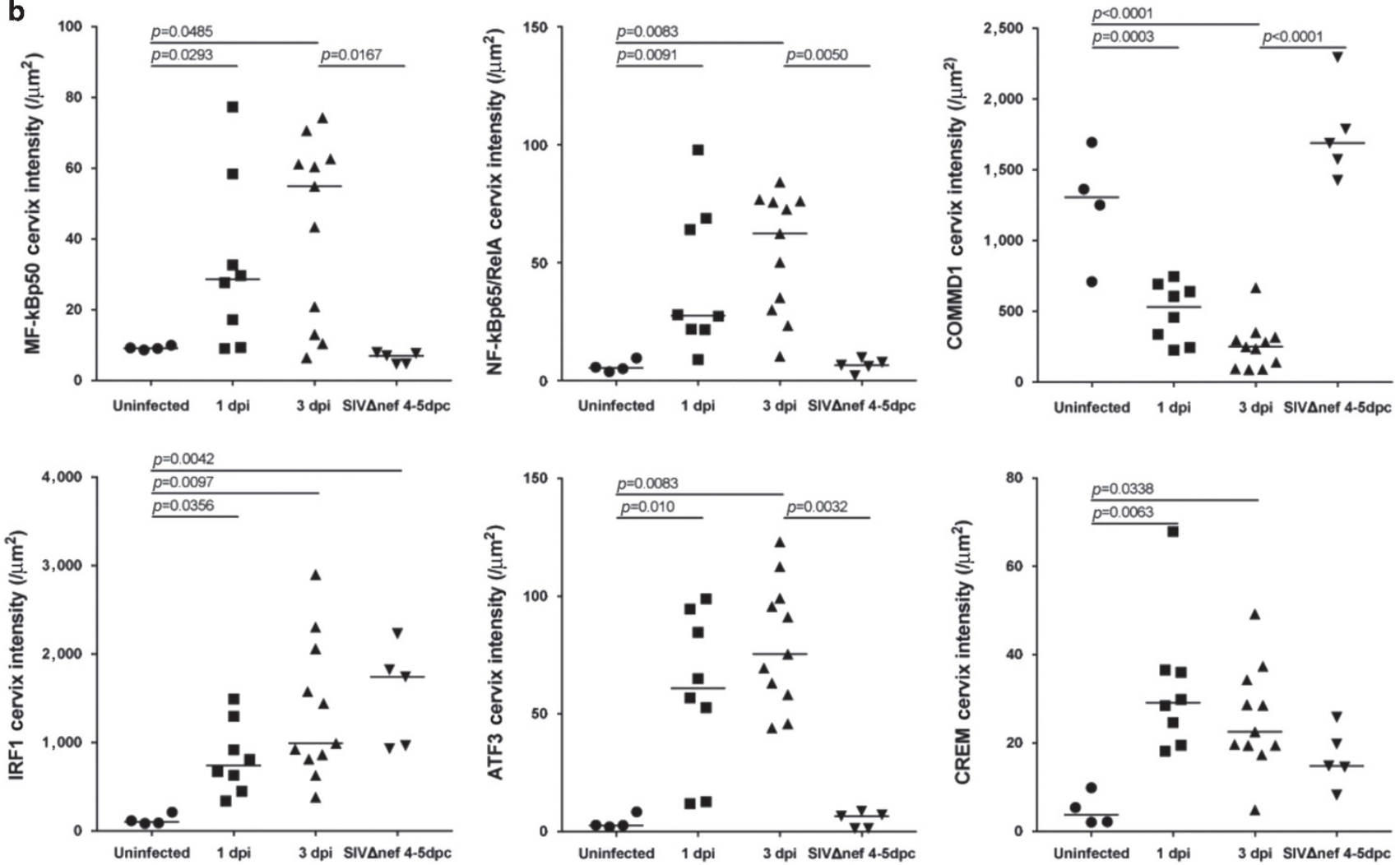

Figure 3 NF-kB signaling pathways activated in vivo in cervical epithelium of naïve but not SIV $\Delta$ nef-vaccinated animals. (a) Increased expression of NF-kBp50 and NF-kBp65/RelA in cervical epithelium and decreased expression of COMMD1 in vivo at days 1 and 3 following vaginal exposure to SIV in naïve animals but not SIV $\Delta$ nef-vaccinated animals ( $4-5 \mathrm{dpc}$ ). Epithelial expression of IRF1, ATF3, and CREM increase in naïve animals following vaginal exposure, as shown ex vivo. In vivo, ATF expression is not increased in vaccinated animals, similar to NF-kB, but IRF1 and CREM expression levels are both elevated in vaccinated animals. (b) Quantification of immunohistochemical epithelial staining.

the microarray results, looking for molecules with relatively global effects on cellular signaling. We identified reduced expression of genes in the GR pathway at $8 \mathrm{~h}$ after exposure of ex vivo cultures to SIV, (Figure 4a), including: (1) up-regulation of GRLF1 (inhibitor of GR expression) and
ZNF653 (repressor of GR transcriptional activity); and (2) down-regulation of DCAF6 (enhancer of GR expression), TTLL5 (GR-regulated gene), NCOA1/3, and NRIP1 (GR coactivators). In vivo, we found the GR dominantly expressed by cervical epithelium in uninfected FRT mucosa 
a
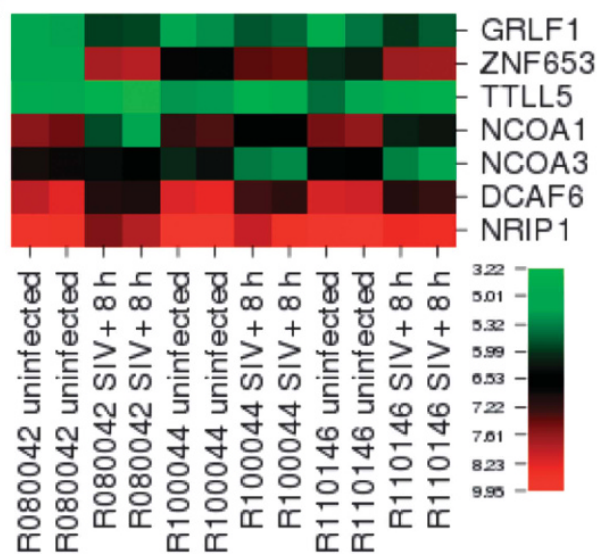

b

Uninfected

$1 \mathrm{dpi}$

$3 \mathrm{dpi}$

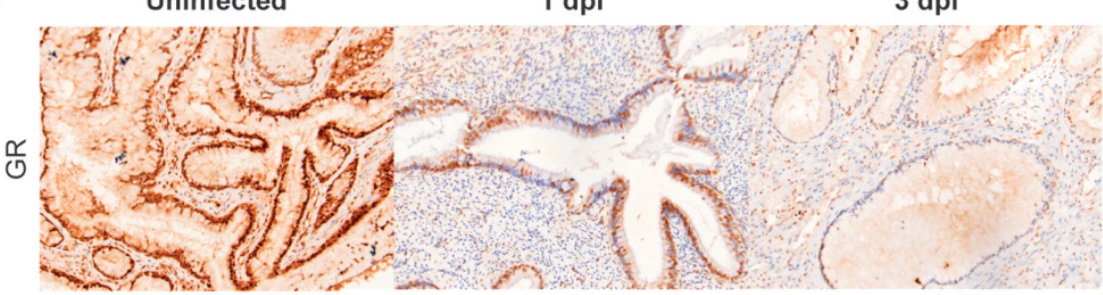

SIV $\Delta$ nef 4-5dpc

SIV $\Delta$ nef $7 \mathrm{dpc}$

$7 \mathrm{dpi}$
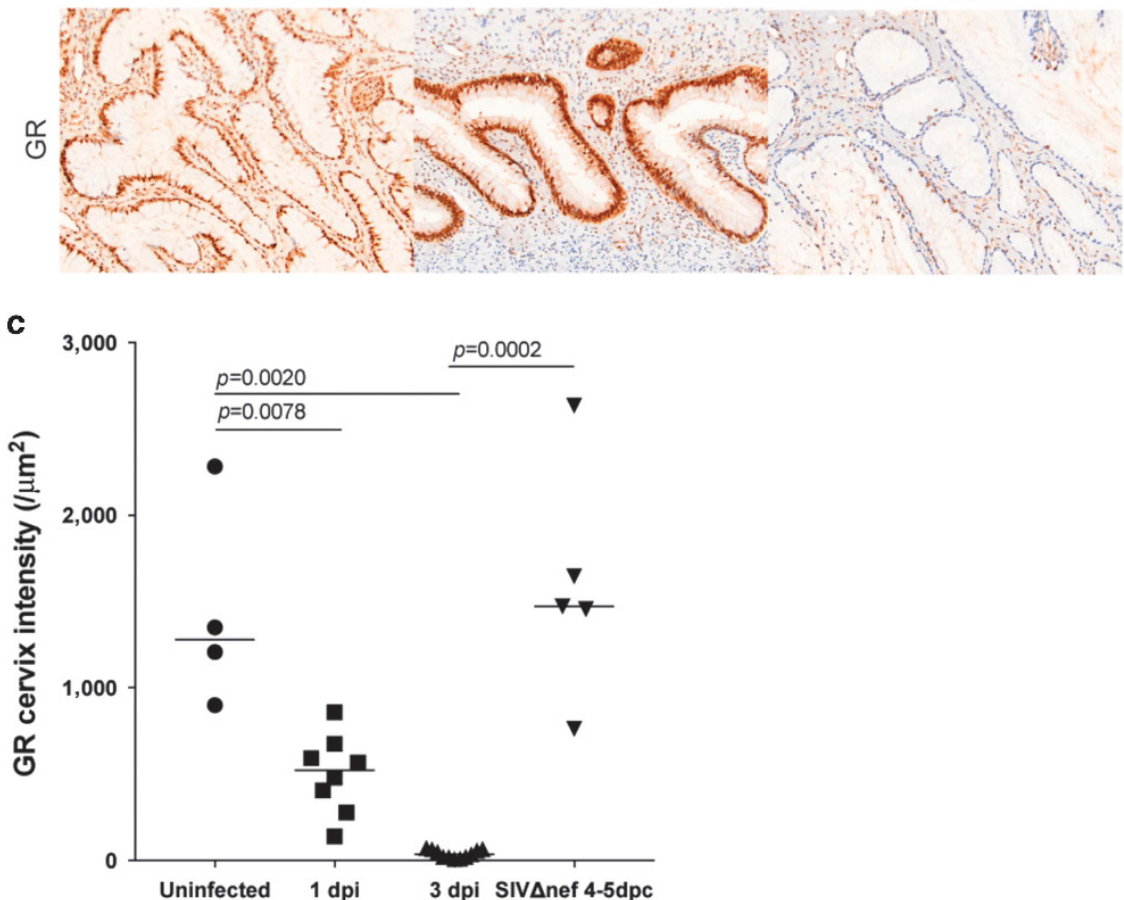

Figure 4 Epithelial glucocorticoid receptor (GR) expression decreases in response to SIV in naïve but not SIV $\Delta$ nef-vaccinated animals. (a) Microarray analysis ex vivo of cervical tissue explants of rhesus macaques after $8 \mathrm{~h}$ topical exposure to SIV. Increases in transcripts for Inhibitors (GRLF1) and repressors (ZNF653) of GR expression and down-regulation of DCAF6 (enhancer of GR expression), TTLL5 (GR-regulated gene), NCOA1/3, and NRIP1 (GR coactivators). (b) In vivo, GR is expressed at a high level mainly in the cervical epithelium of uninfected/unvaccinated animals. Following vaginal challenge, epithelial expression of GR is reduced in unvaccinated animals, but is maintained at high levels in SIV $\Delta$ nef-vaccinated animals through 7 dpc. (c) Quantitative image analysis of GR expression in the cervical tissues of vaccinated and unvaccinated animals before and after vaginal inoculation.

(Figure $4 \mathbf{b}$ ), and the expression notably decreased as early as 1 dpi with continuing decreases at 3 dpi in naïve animals following vaginal challenge with SIV. In sharp contrast, high levels of epithelial GR expression after vaginal challenge were maintained in SIV $\Delta$ nef-vaccinated animals through $7 \mathrm{dpc}$ (Figure 4b,c). 


\section{Virus-immune complexes reverse changes in GR and NF-kB signaling in response to SIV vaginal exposure}

We have previously shown that $\operatorname{SIV} \Delta$ nef vaccination can suppress epithelial responses by SIV-specific IC binding to the inhibitory IgG receptor FCGR2B on cervical epithelium. Here we show that topical application of SIV-specific IC onto the cervical mucosa prior to SIV exposure stabilized GR expression and moderated increases in the epithelial expression of NF-kBp50/p65, which is disrupted by FCGR2B blocking Ab (Figure 5). Thus, the reciprocal changes in the NF-kB and GR signaling could be downstream events after FCGR2B-IC binding that subsequently contribute to its inhibitory effect on the cervical epithelial response to SIV.

\section{DISCUSSION}

A deeper understanding of HIV infection and transmission at mucosal surfaces is important for the development of more effective approaches for prevention. To that end, we have been specifically focusing on the very early events in the cervicovaginal mucosa using the SIV-RM model of HIV-1 sexual transmission to women. In this Non-Human Primate model, our previous work identified induction of chemokines in the cervical epithelium within $1 \mathrm{dpc}$ that leads to CD4 + T-cell recruitment and local expansion of infection as the antecedent to establishing systemic infection. ${ }^{5,6}$ In this study, we pushed the time frame further back using ex vivo cultures to define the immediate epithelial response to exposure to SIV in naive animals and the alterations in those response in SIVmac239 nef-vaccinated animals. The clear picture now emerges that the literal front line of the mucosal immune system is an epithelium endowed with the circuitry and machinery to initiate immune responses mediated by the underlying innate and adaptive immune system. At the same time, the epithelium can balance initial responses through reciprocal systems to launch, a
GR

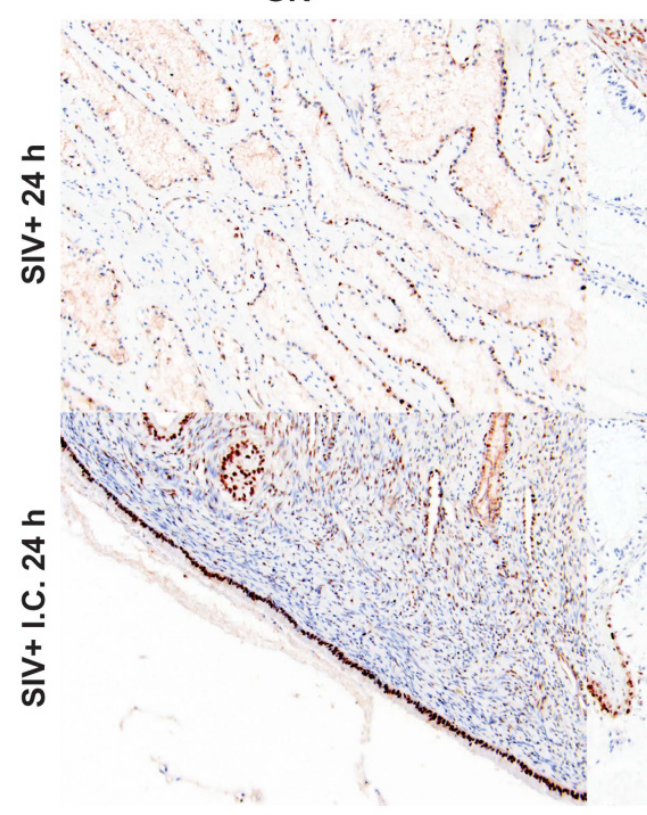

NF-kBp50

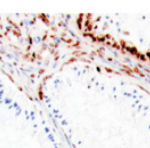

\section{.

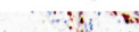

\section{NF-kBp65}
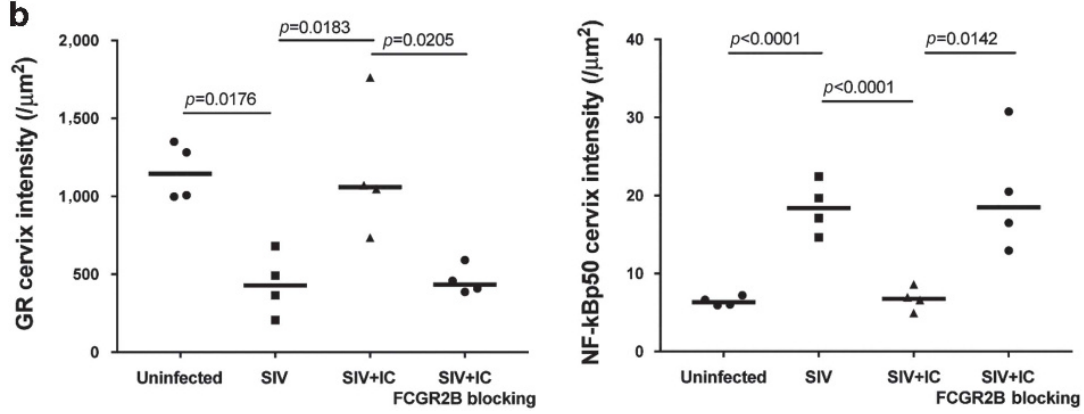

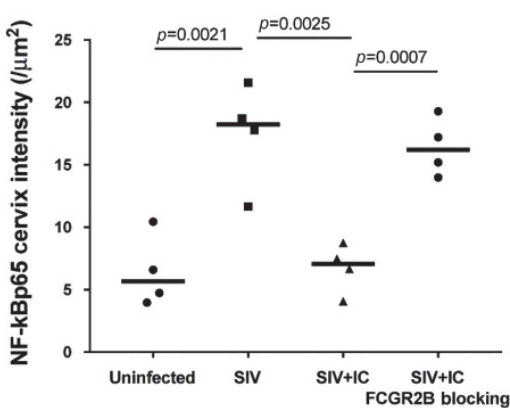

Figure 5 FCGR2B-immune complex (IC)-mediated suppression of epithelial NF-kB and glucocorticoid receptor (GR) signaling responses to SIV. (a) In ex vivo cervical tissues from naïve rhesus macaques, topical exposure to SIV reduces the epithelial expression of GR and induces epithelial expression NF-kB signaling molecules. Topical application of SIV-specific IC onto the cervical epithelium prior to SIV exposure partially suppresses these responses. (b) Quantitative image analysis of immunohistochemical epithelial staining. Fresh cervical tissues from four uninfected rhesus macaques were used. Uninfected and infected tissues from the same animal were compared pairwise to measure the effect of SIV-specific IC-FCGR2B inhibitory signaling on NF-kB vs. GR signaling pathways. Each dot represents a single animal. 
Table 1 Primary Abs used in this study

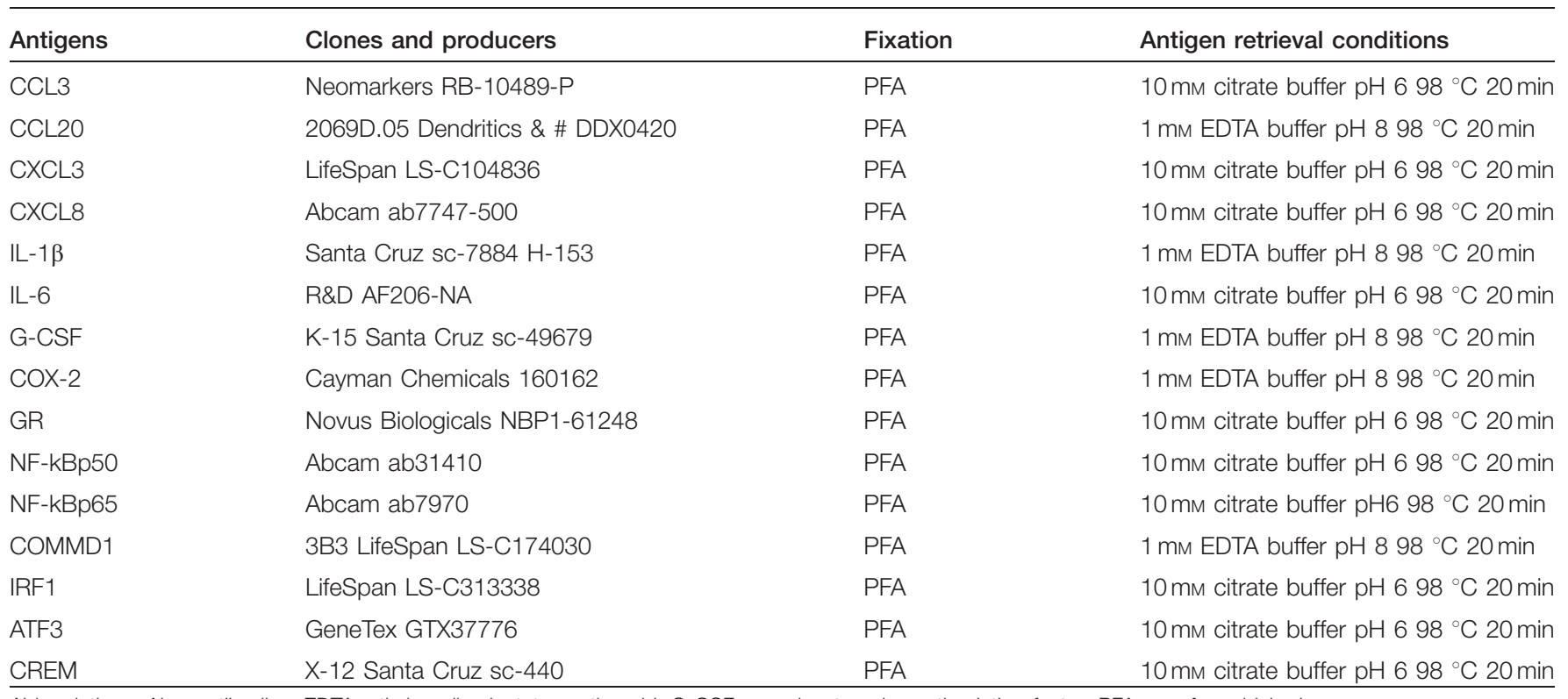

Abbreviations: Abs, antibodies; EDTA, ethylenediaminetetraacetic acid; G-CSF, granulocyte colony-stimulating factor; PFA, paraformaldehyde.

moderate or inhibit pro-inflammatory responses by mechanisms related to prior experience with microbes and antigens.

Here we identify the NF-kB signaling pathway ${ }^{11}$ in the epithelium as a master regulatory component to initiate innate and adaptive immune responses and inflammatory activation at mucosal front lines after SIV exposure. Indeed, besides inflammatory cytokines such as IL-1b, the majority of the genes whose expression increases in the epithelium in response to SIV exposure are also targets of NF-kB activation. The reciprocal coordinate regulation of GR and NF-kB expression in the epithelium is consistent with a check-and-balance system in the FRT mucosa to maintain homeostatic balance and avert the pathological consequences of sustained inflammation until a pathogen is encountered. Mucosal exposure to SIV inoculum then rapidly reduces GR expression and increases NF-kB expression to initiate a pro-inflammatory state and immune response. Paradoxically, this response actually facilitates transmission through recruitment of $\mathrm{CD} 4 \mathrm{~T}$ cell targets to amplify infection at the portal of entry.

Perhaps also paradoxically and counter-intuitively, SIVmac239 $\Delta$ nef vaccination inhibits the inflammatory and immune response to vaginal challenge. We now show that vaccination prevents the reciprocal changes of GR and NF-kB expression in mucosal epithelium after challenge, thereby blocking inflammatory activation in the epithelium and mucosal tissues. We provide evidence here that the dampening effects of SIVmac239 $\Delta$ nef vaccination on NF-kB signaling and FRT inflammation may be mediated by IC interactions with FCGR2B. Previous exposure to SIV during the vaccination elicits high concentrations of virus-specific Abs at mucosal border that form IC with incoming virus of challenge and subsequently activate the inhibitory signaling through binding to FCGR2B on mucosal epithelium., ${ }^{4,8}$ This vaccine-induced inhibitory mechanism illustrates how the epithelium can provide information and appropriate responses that distinguish between a pathogen that has or has not been previously encountered.

Glycerol monolaurate protection against high-dose vaginal challenge may be mediated by moderating inflammatory activation in the FRT mucosa. We have previously shown that daily vaginal treatment of rhesus macaques with $5 \%$ glycerol monolaurate gel can prevent inflammatory responses in the FRT mucosa after vaginal challenge and completely protect monkeys from repeated intra-vaginal challenges of high-dose SIV. ${ }^{3}$ Moderating the transmission-facilitating responses that increase target cells for local expansion is thus a common theme in the successes of glycerol monolaurate and SIVmac239 $\Delta$ nef vaccination in protection against stringent high-dose vaginal SIV challenges. ${ }^{3}$ Thus, as a general principle, strategies that similarly target inflammatory pathways could complement other direct approaches of prevention such as topical anti-retroviral drug-based approaches. Given the lower efficacy of drugs in a setting of inflammation in high-risk populations, moderating inflammation and target cell availability could enhance the effectiveness of ART in preventing transmission.

\section{METHODS}

Tissues from SIV-infected animals. New tissue sections were cut for the studies described here from archived genital tissues from previous longitudinal studies of mucosal transmission following SIV high-dose vaginal infection in unvaccinated and SIVmac239 $\Delta$ nef-vaccinated rhesus macaques. ${ }^{4,5}$ In brief, in those studies, monkeys had been inoculated intravaginally with pathogenic SIVmac251 twice in a single day, with a 4 -h interval between inoculations. Each inoculation contained $1 \mathrm{ml}$ virus stock of $10^{5}$ TCID50. Fresh tissues obtained at necropsy were fixed in $4 \%$ paraformaldehyde or SAFEFIX II (Fisher Scientific, Kalamazoo, MI, USA), and embedded in paraffin as previously reported. ${ }^{6}$ In the cohort of SIV high-dose vaginal infection, 
necropsy was carried out at prior to challenge (uninfected controls $n=4), 1(n=8)$, and $3(n=11)$ days post-inoculation (dpi). In the SIV $\Delta$ nef vaccine study, animals had been vaccinated 20 weeks before vaginal challenge. Vaccinated animals were necropsied at $4(n=3), 5$ $(n=2)$, and $7(n=2)$, days post-challenge (dpc).

Ex vivoculture system. The entire fresh cervical tissue from a healthy, uninfected, young adult rhesus macaque was equally split into two pieces and embedded in agarose gel, leaving only the epithelium surfaces exposed upward. Virus stocks or medium (uninfected control) were topically applied upon epithelium surfaces. After an incubation of $8 \mathrm{~h}$ at $37^{\circ} \mathrm{C}$, each piece of cervical tissue was further divided. Half was fixed in paraformaldehyde and embedded in paraffin for immunohistochemical tissue mapping. On the other half, the surface layer of $1-2 \mathrm{~mm}$ in depth was carefully collected and immediately frozen for microarray analysis. Cervical tissues from 3 rhesus macaques were infected, cultured, and processed in this study.

A similar ex vivo system was used to examine the effects of SIVspecific IC on early cervicovaginal epithelium responses to SIV exposure. The fresh cervical tissue was equally split into three pieces and then topically applied with medium (uninfected control), SIV, and SIV plus IC that were prepared by mixing SIV virus with rhesus antigp41 mAb 4.9C or anti-SIV serum. After $24 \mathrm{~h}$ incubation, the tissues were fixed or frozen, and then compared pairwise among tissues from the same animal individual for IC-induced changes in cervical mucosa. For immune complex formation, $1.2 \times 10^{5}$ infectious units of SIVmac25 were combined with excess $(\sim 7 \mu \mathrm{g})$ antibody (gp41 monoclonal antibody $4.9 \mathrm{C}$ or serum from (SIV $\Delta$ nef)-vaccinated animals) and incubated for $60 \mathrm{~min}$ at room temperature before application to explants. For blocking Fc $\gamma$ RIIb, explants were preincubated with $5 \mu \mathrm{g}$ Fc $\gamma$ RIIb blocking Abs (Abcam, catalog \# ab45143, rabbit mAb, lot \# GR32165-2; Abnova, catalog \# H00002213-B01P, mouse pAb, lot \# 08338 WULZ) for $30 \mathrm{~min}$ at $37^{\circ} \mathrm{C} / 5 \% \mathrm{CO}_{2}$ before addition of immune complexes.

Microarray analysis. The global transcription analysis was performed on the Affymetrix Rhesus Gene 1.0 ST Array platform (Affymetrix, Santa Clara, CA, USA). RNA extractions, synthesis of labeled complementary RNA probes, microarray hybridization, and data collection followed previously published procedures. ${ }^{12}$ Preparation of cRNA probes and microarray hybridizations were done in duplicate for each RNA sample. The raw microarray data from the "CEL" files were processed in " $R$ ". In brief, CEL files from individual hybridizations were processed using the robust multi-chip average algorithm to perform global background correction, quantile normalization, and probeset summarization. The expression levels from duplicate microarrays of the same RNA sample were correlated and averaged. Tests for variations of gene expression before and after infection across pairwise tissues of the three animals were conducted using the nonparametric Wilcoxon signed-rank test. Fold changes in gene expression (logarithm transformed intensities) before and after infection were calculated with the ratio of the means. After statistical analysis, genes were selected based on their associated $P$-values $(<0.05)$, false positive cut-off $q$-values $(<0.2)$, and fold changes in expression $(>1.2)$. Significantly changed genes and transcripts were uploaded into NetAffix Analysis Center (http://www.affymetrix.com/ analysis/index.affx) to query gene ontology information and into Ingenuity Pathways Analysis (Ingenuity Systems, www.ingenuity.com) for gene annotation and pathway analysis.

Quantitative real-time PCR. Altered expression of genes detected in the microarray analysis was confirmed by quantitative real-time PCR performed on total RNA using an ABI-PRISM 7900HT Sequence Detection System and SYBR green PCR Master Mix (Applied Biosystems, Foster City, CA). The cDNA was synthesized from 200 to $1,000 \mathrm{ng}$ total RNA. Each primer pair for quantitative PCR was tested with a logarithmic dilution of cDNA to generate a standard curve, which was used to calculate the starting quantity of target RNA.
Primers specific for GAPDH were used as an endogenous standard to normalize samples. Fold-change was calculated by dividing the normalized post-infected sample quantity with the normalized preinfected control quantity.

Immunohistochemistry and quantitative image analysis. Immunohistochemical staining and quantitative image analysis were performed as described elsewhere. ${ }^{6}$ The primary Abs used in this study are summarized in Table 1. In brief, tissue sections were deparaffinized in xylene and rehydrated in phosphate-buffered saline. After blocking in Background Sniper (Biocare Medical, Concord, CA, USA), sections were incubated with primary Abs at $4{ }^{\circ} \mathrm{C}$ overnight. Then signals were amplified with $2^{\circ} \mathrm{Ab}$-Biotin $+\mathrm{ABC}$ Kit (Vector Lab, Burlingame, CA, USA). Nuclei were counterstained with hematoxylin (Invitrogen, Eugene, OR, USA). For each tissue block, 2-3 sections at various depths were cut and stained for specific immunohistochemistry markers. Images were taken on Olympus BX60 microscope (Olympus, Center Valley, PA, USA). For quantitative image analysis, stained tissue sections were scanned by the Aperio ScanScope System (Leica, Buffalo Grove, IL, USA). The entire endocervical tissues were then analyzed and quantified by the ImageScope software (Leica) to measure the pixel intensities, numbers of positive cells and nuclei, and tissue areas, using the pixel intensity, membrane functions, and nuclei counting functions provided by the software. We did not select particular areas for quantification in this work. For a specific immunohistochemistry marker, all animals were stained, quantified, and included in the plots. Each dot in quantification plots of specific immunohistochemistry markers represents an individual animal showing the mean value of all sections. Due to space limit, only representative images of immunohistochemistry staining were shown in the figures.

Statistical tests. The non-parametric Mann-Whitney test was used to compare the variations in expression of immunohistochemical markers over the course of infection. Statistical analyses were carried out using Prism 4 software.

\section{ACKNOWLEDGMENTS}

We thank R. Desrosiers and C. Miller for virus stocks; C. Miller for tissue samples from uninfected animals and from unvaccinated and infected animals; A. Carville for expert veterinary care; E. Curran and A. Miller for assistance with tissue processing and analysis; C. O'Neill and T. Leonard for help in preparing the manuscripts and figures. This work was supported by the International AIDS Vaccine Initiative, National Institutes of Health grants Al071306 and RR00168, and in part with federal funds from the National Cancer Institute, National Institutes of Health, under contract HHSN261200800001E.

\section{AUTHOR CONTRIBUTIONS}

L.S. and A.T.H. designed this study; L.S., C.S.R., and A.J.S. carried out microarray analysis and ex vivo explant experiments; L.S., L.D., K.E.P., and S.W. carried out immunohistochemistry analysis; M.Z. processed tissues; P.J.S. participated in ex vivo explant experiment; R.P.J. carried out animal work of live attenuated virus vaccine SIV vaccination; J.V.C. facilitated manuscript preparation.

\section{DISCLOSURE}

The authors declared no conflict of interest.

(c) 2018 Society for Mucosal Immunology

\section{REFERENCES}

1. UNAIDS 2013 Report on the global AIDS epidemic, available at http:// www.unaids.org/sites/default/files/media_asset/UNAIDS_Global_Report _2013_en_1.pdf (2013).

2. Quinn, T.C. \& Overbaugh, J. HIV/AIDS in women: an expanding epidemic. Science 308, 1582-1583 (2005).

3. Li, Q. et al. Glycerol monolaurate prevents mucosal SIV transmission. Nature 458, 1034-1038 (2009). 


\section{ARTICLES}

4. Li, Q. et al. Live simian immunodeficiency virus vaccine correlate of protection: local antibody production and concentration on the path of virus entry. J. Immunol. 193, 3113-3125 (2014).

5. Miller, C.J. et al. Propagation and dissemination of infection after vaginal transmission of simian immunodeficiency virus. J. Virol. 79, 9217-9227 (2005).

6. Shang, L. et al. Epithelium-innate immune cell axis in mucosal responses to SIV. Mucosal Immunol. 10, 508-519 (2016).

7. Shang, L. et al. NK cell responses to simian immunodeficiency virus vaginal exposure in naive and vaccinated rhesus macaques. J. Immunol. 193, 277-284 (2014).

8. Smith, A.J. et al. Live simian immunodeficiency virus vaccine correlate of protection: immune complex-inhibitory fc receptor interactions that reduce target cell availability. J. Immunol. 193, 3126-3133 (2014).

9. Zeng, M. et al. Mucosal humoral immune response to SIVmac239nef vaccination and vaginal challenge. J. Immunol. 196, 2809-2818 (2016).

10. Adnan, S. et al. Persistent low-level replication of SIVdeltanef drives maturation of antibody and CD8 T cell responses to induce protective immunity against vaginal SIV Infection. PLoS Pathog. 12, e1006104 (2016).

11. Altfeld, M. \& Gale, M. Jr. Innate immunity against HIV-1 infection. Nat. Immunol. 16, 554-562 (2015).

12. Smith, A.J. et al. Host genes associated with $\mathrm{HIV}-1$ replication in lymphatic tissue. J. Immunol. 185, 5417-5424 (2010). 